\title{
A Mechanism-Design Approach to Speculative Trade*
}

\author{
Kfir Eliaz ${ }^{\dagger}$ and Ran Spiegler ${ }^{\ddagger}$
}

November 29, 2006

\section{Introduction}

One of the primary tasks of the mechanism-design literature has been to draw theoretical barriers to trade due to asymmetric information. However, this literature has focused exclusively on trade that is motivated by differences in tastes. In principle, one could pose the same set of questions when the motivation for trade is differences in prior beliefs. What are the limits on the ability to realize gains from speculative trade, when the agents' priors are not common knowledge? What are the mechanisms that enable agents to realize these gains? These questions have been neglected by the literature, mostly because of the ubiquity of the common-prior assumption in economic modeling. In this note, we examine an environment in which agents have different prior beliefs regarding a state of nature that may affect the future actions of one of them. This creates a motive among the agents to bet on the future outcome. However, we assume that the agents' priors are private information. We apply a mechanism-design approach in order to examine the extent to which this form of asymmetric information creates a barrier to speculative bets. ${ }^{1}$

${ }^{*}$ We are grateful to Larry Samuelson (co-editor) for helping us improve the paper. We also thank Barton Lipman, Eric Maskin, Ady Pauzner, Wolfgang Pesendorfer, Ariel Rubinstein and especially Eddie Dekel, for helpful conversations and comments. Financial support from the US-Israel Binational Science Foundation, Grant No. 2002298 is gratefully acknowledged.

${ }^{\dagger}$ Dept. of Economics, NYU. 269 Mercer St., New York, NY 10003, E-mail: kfir.eliaz@nyu.edu, URL: http://homepages.nyu.edu/ ${ }^{\mathrm{ke}} 7$

${ }_{\ddagger}^{\ddagger}$ Department of Economics, University College London, Gower St., London WC1E 6BT. E-mail: r.spiegler@ucl.ac.uk. URL: http://www.homepages.ucl.ac.uk/ uctprsp

${ }^{1}$ Other authors have pointed out that asymmetric information may inhibit speculative trade, even in the presence of non-common priors. Morris (1994) provides necessary and sufficient conditions for no-trade results to persist in such environments. Chung and Ely (2005) study auction design in such an environment, and show that incentive-compatibility constraints may exclude mutually beneficial bets from the revenue-maximizing mechanism. 
We demonstrate our approach with a simple two-period example. In period 2, an agent faces a decision problem whose payoff structure depends on an unverifiable state of nature. The agent knows the state in period 2, but in period 1 the state is still unknown; the agent and another party, called a "speculator", hold different prior beliefs and therefore have a motive to bet. Since the state is unverifiable, the parties cannot bet on its realization. However, they can bet on the agent's second-period action, which is verifiable. A bet is a function that assigns budget-balanced transfers to each action. The parties' priors are private information, but it is common knowledge that they are independently drawn from a distribution $F$. We define a notion of "constrained interim-efficient" (CIE) bets and ask whether they can be implemented in Bayesian equilibrium by some mechanism.

In this model, the parties bet on an outcome that can be manipulated by the agent. This feature fits many real-life situations. For instance, debt contracts can sometimes be viewed as bets between over-optimistic entrepreneurs and more realistic creditors over the profitability of a project (see Landier and Thesmar (2005)). Such bets are manipulable because the entrepreneur may prefer to risk bankruptcy and persist in developing the project even after receiving bad news about its profitability. Similarly, when a fashion retailer and a supplier sign a backup agreement (the supplier commits to provide a minimal quantity for the coming season, while the retailer commits to pay a penalty if his order falls short of this quantity), they are essentially betting on the level of seasonal demand (see Bazerman and Gillespie (1999)). This bet is manipulable because the retailer may have an incentive to submit an order in excess of the true demand, in order to avoid the penalty. Finally, when a monopolistic commodity supplier trades with speculators in a derivative market associated with the commodity, he essentially bets on the commodity's future price. However, the supplier can manipulate the market price through his supply policy, and his incentive to do so depends on the position he took in the derivative market (see Newbery (1984)). ${ }^{2}$

In such environments, a bet is sustainable only if its stakes do not exceed the agent's cost of manipulating the bet's outcome. But this means that potential gains from speculative bets are bounded. A CIE bet in such an environment maximizes these gains, subject to the constraint that the agent does not want to manipulate its outcome. The bounds on the stakes of bets in our model are thus endogenous. This allows us to establish a link between the implementability of CIE bets and the payoff structure of the underlying decision problem. Our main result is that CIE bets can be

\footnotetext{
${ }^{2}$ Of course, in reality these situations are characterized by asymmetric information as well as heterogeneous prior beliefs. We abstract from the former and focus entirely on the latter.
} 
implemented for a larger set of distributions $F$ when the agent's costs of manipulating the bet's outcome become more even across states. In particular, when they are equal, implementation is possible for any $F$. In contrast, when unilateral manipulation is costless in one state, implementation is impossible for all $F$.

The technical basis for this result is a formal analogy to a conventional mechanismdesign model due to Cramton, Gibbons and Klemperer (1987) - CGK henceforth which extends the buyer-seller framework of Myerson and Satterthwaite (1983) to more general ownership structures, namely "partnerships". Implementing CIE bets turns out to be analogous to dissolving a partnership efficiently. The analogy is not merely formal; we believe that it actually provides insight into the phenomenon of speculative trade. A manipulable bet is like an asset - an entitlement to receive a prize conditional on a random event - whose value and initial ownership structure are determined by the underlying manipulation costs.

This work follows up Eliaz and Spiegler (2005,2006), in which we analyze profitmaximizing contract design by a monopolist who faces consumers with diverse abilities to forecast their future tastes. In Eliaz and Spiegler (2006), consumer preferences are dynamically inconsistent, and consumer types differ in their prior belief that their tastes will not change. Eliaz and Spiegler (2005) analyze a similar problem with dynamically consistent preferences. In both cases, non-common priors are necessary for price discrimination.

\section{The Model}

Consider an agent who faces a choice between two actions: $a$ or $b$. His payoff from each action depends on the state of nature. There are two possible states. The agent's von Neumann-Morgenstern utility function is $u$ in one state and $v$ in the other. With slight abuse of notation, we denote states by the utility functions that characterize them, given by the following table:

$\begin{array}{ccc}\text { action } \backslash \text { state } & u & v \\ a & A & D \\ b & C & B\end{array}$

where $A \geq C$ and $B \geq D$, with at least one strict inequality. The agent privately learns the state of nature before making his decision. A period before the realization of the state, the agent and another party, referred to as a "speculator", hold different prior 
beliefs. Let $\theta_{1}$ and $\theta_{2}$ be the prior probability assigned to state $u$ by the speculator and the agent, respectively.

Because the parties have different priors, they find it mutually beneficial to bet. Since the state is privately observed by the agent, they are unable to bet on it directly. Instead, they can bet on the agent's action, which is verifiable. ${ }^{3}$ We refer to the period in which the state is realized and the action is taken as period 2. The period in which the bet is negotiated is referred to as period 1 . A bet $t:\{a, b\} \rightarrow \mathbb{R}$ is a function that assigns to each action a transfer from the agent to the speculator. A signed bet $t$ modifies the decision problem faced by the agent. His utility from an action $x$ in state $\omega \in\{u, v\}$ is $\omega(x)-t(x)$; the speculator's utility from $x$ is $t(x)$, regardless of the state. If no bet is signed, the agent faces the "bare" decision problem and the speculator receives nothing.

Consider a bet $t$, and suppose that both parties expect that the agent's actions in states $u$ and $v$ will be $x^{u}$ and $x^{v}$. Denote $\mathbf{x}=\left(x^{u}, x^{v}\right)$. Then, the speculator's interim expected payoff from $(\mathbf{x}, t)$ is $\theta_{1} t\left(x^{u}\right)+\left(1-\theta_{1}\right) t\left(x^{v}\right)$, while the agent's is $\theta_{2}\left[u\left(x^{u}\right)-t\left(x^{u}\right)\right]+\left(1-\theta_{2}\right)\left[v\left(x^{v}\right)-t\left(x^{v}\right)\right]$. The term "interim" is fitting because it refers to the parties' expected payoffs upon learning their prior, which is drawn from a common distribution. Note that the sum of the parties' interim expected payoffs can be conveniently written as

$$
\theta_{2} u\left(x^{u}\right)+\left(1-\theta_{2}\right) v\left(x^{v}\right)+\left(\theta_{1}-\theta_{2}\right)\left[t\left(x^{u}\right)-t\left(x^{v}\right)\right]
$$

If the agent could commit to play $x^{u} \neq x^{v}$, there is no upper bound on the stakes of the bet that the parties would want to sign: if $\theta_{1}>(<) \theta_{2}$, they would set $t\left(x^{u}\right) \gg$ $(\ll) t\left(x^{v}\right)$. However, because the agent cannot commit, the parties must take into account his ability to manipulate the bet's outcome. For instance, suppose that the parties agree on a bet that satisfies $t(b)-t(a)>B-D$. Then, the agent will prefer to choose $a$ not only in state $u$ but also in state $v$, because the amount he saves in side payments outweighs the "bare" loss from taking the wrong action. But if the agent's action is the same in both states, the parties cannot benefit from betting on it. Thus, in order to be sustainable, the bet must provide the agent with incentives to take different actions in different states.

A pair $(\mathbf{x}, t)$ is constrained interim-efficient (CIE) if it maximizes (1) subject to

\footnotetext{
${ }^{3}$ Because of the balanced-budget restriction, the parties cannot circumvent the verifiability constraint by playing some general message game in period 2 .
} 
the constraints:

$$
\begin{aligned}
& u\left(x^{u}\right)-t\left(x^{u}\right) \geq u\left(x^{v}\right)-t\left(x^{v}\right) \\
& v\left(x^{v}\right)-t\left(x^{v}\right) \geq v\left(x^{u}\right)-t\left(x^{u}\right)
\end{aligned}
$$

We say that $t$ is a $C I E$ bet if there exists $\mathbf{x}$ such that $(\mathbf{x}, t)$ is CIE. We refer to the expression (1), evaluated at a CIE pair $(\mathbf{x}, t)$, as the CIE surplus. ${ }^{4}$

It follows from (1) that if $\theta_{1}>\theta_{2}$, the parties would want to set $t\left(x^{u}\right)-t\left(x^{v}\right)$ to be equal to the upper bound implied by $(2), u\left(x^{u}\right)-u\left(x^{v}\right)$. In contrast, if $\theta_{1}<\theta_{2}$, they would want to set $t\left(x^{u}\right)-t\left(x^{v}\right)$ to be equal to the lower bound implied by (2), $v\left(x^{u}\right)-v\left(x^{v}\right)$. Both bounds on $t\left(x^{u}\right)-t\left(x^{v}\right)$ are relaxed to their utmost when $x^{u}=a$ and $x^{v}=b$. Thus, we have the following characterization.

Remark 1 A pair $(\mathbf{x}, t)$ is CIE if and only if the following two conditions hold:

(i) $\mathbf{x}$ is ex-post efficient - i.e., $\left(x^{u}, x^{v}\right)=(a, b)$.

(ii) $t$ satisfies:

$$
t(a)-t(b)=\left\{\begin{array}{ccc}
A-C & \text { if } & \theta_{1}>\theta_{2} \\
D-B & \text { if } & \theta_{1}<\theta_{2}
\end{array}\right.
$$

Thus, CIE bets never cause the agent to take a sub-optimal action, and their stakes are determined by his cost of manipulating their outcome.

\section{The Main Result}

We now turn to the question of whether the CIE surplus can be implemented, when the parties' priors are not common knowledge. We assume that each party privately and independently draws his prior on $u$ from a distribution $F$ with support $[0,1]$ and positive continuous density. We consider the problem of implementing the CIE surplus via a direct mechanism. That is, the parties play a two-period game. In period 1, each party $i$ submits a report $\hat{\theta}_{i} \in[0,1]$ (interpreted as his stated prior on $u$ ), or chooses not to participate. If at least one party chooses the latter, there is no bet and the agent faces the "bare" decision problem. If both parties choose to participate, every pair of reports $\hat{\boldsymbol{\theta}}=\left(\hat{\theta}_{1}, \hat{\theta}_{2}\right)$ is assigned a bet $t(\hat{\boldsymbol{\theta}})$, which is disclosed to the agent. In period 2 , after the state of nature is realized, the agent chooses an action $x$ and pays $t(x \mid \hat{\boldsymbol{\theta}})$ to the speculator. In each state $\omega \in\{u, v\}$, he chooses $x$ to maximize $\omega(x)-t(x \mid \hat{\boldsymbol{\theta}})$. Let

\footnotetext{
${ }^{4}$ The welfare criterion employed here is interim Pareto efficiency: for any non-CIE $(\mathbf{x}, t)$, there is another pair $\left(\mathbf{x}^{\prime}, t^{\prime}\right)$ which satisfies (2) and yields higher interim expected utility for both parties.
} 
$\mathbf{x}(\hat{\boldsymbol{\theta}}) \equiv\left(x^{u}(t(\hat{\boldsymbol{\theta}})), x^{v}(t(\hat{\boldsymbol{\theta}}))\right)$ denote the agent's second-period strategy, given $t(\hat{\boldsymbol{\theta}})$. We identify a direct mechanism with the function $t(\hat{\boldsymbol{\theta}})$.

Define $T_{i}^{u}\left(\hat{\theta}_{i}\right) \equiv E_{\theta_{j}} t\left(x^{u} \mid \hat{\theta}_{i}, \theta_{j}\right)$ and $T_{i}^{v}\left(\hat{\theta}_{i}\right) \equiv E_{\theta_{j}} t\left(x^{v} \mid \hat{\theta}_{i}, \theta_{j}\right)$. That is, $T_{i}^{\omega}\left(\hat{\theta}_{i}\right)$ denotes the expected transfer in state $\omega$, given $(\mathbf{x}(\hat{\boldsymbol{\theta}}), t(\hat{\boldsymbol{\theta}}))$, assuming that in period 1 party $i$ reports $\hat{\theta}_{i}$ while party $j$ reports truthfully. Similarly, define $U\left(\hat{\theta}_{2}\right) \equiv E_{\theta_{1}} u\left[x^{u} \mid t\left(\theta_{1}, \hat{\theta}_{2}\right)\right]$ and $V\left(\hat{\theta}_{2}\right) \equiv E_{\theta_{1}} v\left[x^{v} \mid t\left(\theta_{1}, \hat{\theta}_{2}\right)\right]$ to be the agent's expected "bare" payoff in states $u$ and $v$, given $(\mathbf{x}(\hat{\boldsymbol{\theta}}), t(\hat{\boldsymbol{\theta}}))$, assuming that in period 1 he reports $\hat{\theta}_{2}$ while the speculator reports truthfully.

Definition 1 A direct mechanism $t(\hat{\boldsymbol{\theta}})$ implements the CIE surplus for a given distribution $F$ if:

$(E F F)$ There exists a second-period strategy for the agent $\mathbf{x}(\hat{\boldsymbol{\theta}})$ such that whenever $\hat{\boldsymbol{\theta}}=\boldsymbol{\theta}$, the pair $(\mathbf{x}(\hat{\boldsymbol{\theta}}), t(\hat{\boldsymbol{\theta}}))$ is CIE.

(IR) For every $\theta_{1}, \theta_{2}$ :

$$
\begin{aligned}
\theta_{1} T_{1}^{u}\left(\theta_{1}\right)+\left(1-\theta_{1}\right) T_{1}^{v}\left(\theta_{1}\right) & \geq 0 \\
\theta_{2}\left[U\left(\theta_{2}\right)-T_{2}^{u}\left(\theta_{2}\right)\right]+\left(1-\theta_{2}\right)\left[V\left(\theta_{2}\right)-T_{2}^{v}\left(\theta_{2}\right)\right] & \geq \theta_{2} A+\left(1-\theta_{2}\right) B
\end{aligned}
$$

(IC) For every $\theta_{1}, \hat{\theta}_{1}, \theta_{2}, \hat{\theta}_{2}$ :

$$
\begin{aligned}
\theta_{1} T_{1}^{u}\left(\theta_{1}\right)+\left(1-\theta_{1}\right) T_{1}^{v}\left(\theta_{1}\right) & \geq \theta_{1} T_{1}^{u}\left(\hat{\theta}_{1}\right)+\left(1-\theta_{1}\right) T_{1}^{v}\left(\hat{\theta}_{1}\right) \\
\theta_{2}\left[U\left(\theta_{2}\right)-T_{2}^{u}\left(\theta_{2}\right)\right]+\left(1-\theta_{2}\right)\left[V\left(\theta_{2}\right)-T_{2}^{v}\left(\theta_{2}\right)\right] & \geq \theta_{2}\left[U\left(\hat{\theta}_{2}\right)-T_{2}^{u}\left(\hat{\theta}_{2}\right)\right]+\left(1-\theta_{2}\right)\left[V\left(\hat{\theta}_{2}\right)-T_{2}^{v}\left(\hat{\theta}_{2}\right)\right]
\end{aligned}
$$

Conditions $I R$ and $I C$ are standard: each party chooses to participate and report his true prior in period 1. Condition EFF is less standard, as it also deals with the agent's post-mechanism behavior. It says that whenever the parties report their priors truthfully in period 1, the agent's second-period strategy and the bet that the mechanism assigns to the first-period profile of reports constitute a CIE pair.

Proposition 1 There exists a distribution $F$ for which the CIE surplus is implementable, if and only if both $A-C>0$ and $B-D>0$. Moreover, as the ratio $\frac{A-C}{B-D}$ becomes closer to one, the set of distributions for which the CIE surplus is implementable expands. When $A-C=B-D$, the CIE surplus is implementable for every distribution $F$.

Proof. By Remark 1, (x( $(\hat{\boldsymbol{\theta}}), t(\hat{\boldsymbol{\theta}}))$ is CIE if and only if $\mathbf{x}(\hat{\boldsymbol{\theta}})=(a, b)$ for every $\hat{\boldsymbol{\theta}}$ and $t(\hat{\boldsymbol{\theta}})$ satisfies (3). In particular, this means that under $(E F F), U\left(\theta_{2}\right)=A$ and $V\left(\theta_{2}\right)=B$ 
for all $\theta_{2}$. Therefore, Definition 1 may be restated as follows. A direct mechanism $t(\hat{\boldsymbol{\theta}})$ implements the CIE surplus for a given distribution $F$ if:

$(E F F)$ Whenever $\hat{\boldsymbol{\theta}}=\left(\theta_{1}, \theta_{2}\right)$,

$$
t\left(a \mid \theta_{1}, \theta_{2}\right)-t\left(b \mid \theta_{1}, \theta_{2}\right)=\left\{\begin{array}{lll}
A-C & \text { if } & \theta_{1}>\theta_{2} \\
D-B & \text { if } & \theta_{1}<\theta_{2}
\end{array}\right.
$$

$(I R)$ For every $\theta_{1}, \theta_{2}$ :

$$
\begin{aligned}
& \theta_{1} E_{\theta_{2}^{\prime}}\left[t\left(a \mid \theta_{1}, \theta_{2}^{\prime}\right)-t\left(b \mid \theta_{1}, \theta_{2}^{\prime}\right)\right]+E_{\theta_{2}^{\prime}} t\left(b \mid \theta_{1}, \theta_{2}^{\prime}\right) \geq 0 \\
& \theta_{2} E_{\theta_{1}^{\prime}}\left[t\left(b \mid \theta_{1}^{\prime}, \theta_{2}\right)-t\left(a \mid \theta_{1}^{\prime}, \theta_{2}\right)\right]-E_{\theta_{1}^{\prime}} t\left(b \mid \theta_{1}^{\prime}, \theta_{2}\right) \geq 0
\end{aligned}
$$

(IC) For every $\theta_{1}, \hat{\theta}_{1}, \theta_{2}, \hat{\theta}_{2}$ :

$$
\begin{aligned}
& \theta_{1} E_{\theta_{2}^{\prime}}\left[t\left(a \mid \theta_{1}, \theta_{2}^{\prime}\right)-t\left(b \mid \theta_{1}, \theta_{2}^{\prime}\right)\right]+E_{\theta_{2}^{\prime}} t\left(b \mid \theta_{1}, \theta_{2}^{\prime}\right) \geq \theta_{1} E_{\theta_{2}^{\prime}}\left[t\left(a \mid \hat{\theta}_{1}, \theta_{2}^{\prime}\right)-t\left(b \mid \hat{\theta}_{1}, \theta_{2}^{\prime}\right)\right]+E_{\theta_{2}^{\prime}} t\left(b \mid \hat{\theta}_{1}, \theta_{2}^{\prime}\right) \\
& \theta_{2} E_{\theta_{1}^{\prime}}\left[t\left(b \mid \theta_{1}^{\prime}, \theta_{2}\right)-t\left(a \mid \theta_{1}^{\prime}, \theta_{2}\right)\right]-E_{\theta_{1}^{\prime}} t\left(b \mid \theta_{1}^{\prime}, \theta_{2}\right) \geq \theta_{2} E_{\theta_{1}^{\prime}}\left[t\left(b \mid \theta_{1}^{\prime}, \hat{\theta}_{2}\right)-t\left(a \mid \theta_{1}^{\prime}, \hat{\theta}_{2}\right)\right]-E_{\theta_{1}^{\prime}} t\left(b \mid \theta_{1}^{\prime}, \hat{\theta}_{2}\right)
\end{aligned}
$$

Our method of proof is to establish a formal equivalence between this definition of implementation of the CIE surplus and the problem of efficiently dissolving a partnership. This latter problem is defined as follows. A two-member partnership is a triple $\left\langle r_{1}, r_{2}, F\right\rangle$, where $r_{i}$ is partner $i$ 's initial share in a jointly owned asset and $F$ is the continuous distribution on $[0,1]$ from which both partners independently (and privately) draw their valuations of the asset. The partners are assumed to be risk-neutral with quasi-linear preferences, where $\theta_{i}$ denotes partner $i$ 's value for a unit of the asset. A partnership is dissolved efficiently if the entire asset $r_{1}+r_{2}$ is allocated to the partner with the highest valuation. A direct mechanism for dissolving a partnership is a pair of functions $(q(\hat{\boldsymbol{\theta}}), m(\hat{\boldsymbol{\theta}}))$ that assign, for each pair of reported values $\hat{\boldsymbol{\theta}}$, an allocation of shares, $q_{1}(\hat{\boldsymbol{\theta}})$ and $q_{2}(\hat{\boldsymbol{\theta}})$, and a pair of monetary transfers, $m_{1}(\hat{\boldsymbol{\theta}})$ and $m_{2}(\hat{\boldsymbol{\theta}})$, such that for all $\hat{\boldsymbol{\theta}}, q_{i}(\hat{\boldsymbol{\theta}}) \geq 0, q_{1}(\hat{\boldsymbol{\theta}})+q_{2}(\hat{\boldsymbol{\theta}})=r_{1}+r_{2}$ and $m_{1}(\hat{\boldsymbol{\theta}})+m_{2}(\hat{\boldsymbol{\theta}})=0$. A mechanism $(q(\hat{\boldsymbol{\theta}}), m(\hat{\boldsymbol{\theta}}))$ efficiently dissolves a partnership $\left\langle r_{1}, r_{2}, F\right\rangle$ if it satisfies the following properties for $i=1,2$ :

$\left(E F F^{*}\right)$ Whenever $\hat{\boldsymbol{\theta}}=\boldsymbol{\theta}$,

$$
q_{i}(\hat{\boldsymbol{\theta}})=\left\{\begin{array}{ccc}
r_{1}+r_{2} & \text { if } & \hat{\theta}_{i}>\hat{\theta}_{j} \\
0 & \text { if } & \hat{\theta}_{i}<\hat{\theta}_{j}
\end{array}\right.
$$

$\left(I R^{*}\right)$ For every $i=1,2$ and every $\theta_{i}: \quad \theta_{i} Q_{i}\left(\theta_{i}\right)+M_{i}\left(\theta_{i}\right) \geq \theta_{i} r_{i}$ 
$\left(I C^{*}\right)$ For every $i=1,2$ and every $\theta_{i}, \hat{\theta}_{i}: \quad \theta_{i} Q_{i}\left(\theta_{i}\right)+M_{i}\left(\theta_{i}\right) \geq \theta_{i} Q_{i}\left(\hat{\theta}_{i}\right)+M_{i}\left(\hat{\theta}_{i}\right)$

where $Q_{i}\left(\hat{\theta}_{i}\right) \equiv E_{\theta_{j}} q_{i}\left(\hat{\theta}_{i}, \theta_{j}\right)$ and $M_{i}\left(\hat{\theta}_{i}\right) \equiv E_{\theta_{j}} m_{i}\left(\hat{\theta}_{i}, \theta_{j}\right)$.

Define:

$$
\begin{aligned}
r_{1} & =B-D \\
r_{2} & =A-C \\
q_{1}(\hat{\boldsymbol{\theta}}) & \equiv B-D+t(a \mid \hat{\boldsymbol{\theta}})-t(b \mid \hat{\boldsymbol{\theta}}) \\
q_{2}(\hat{\boldsymbol{\theta}}) & \equiv A-C-t(a \mid \hat{\boldsymbol{\theta}})+t(b \mid \hat{\boldsymbol{\theta}}) \\
m_{1}(\hat{\boldsymbol{\theta}}) & \equiv t(b \mid \hat{\boldsymbol{\theta}})
\end{aligned}
$$

By simple algebra, we can see that $(E F F),(I R)$ and $(I C)$ hold if and only if $\left(E F F^{*}\right)$, $\left(I R^{*}\right)$ and $\left(I C^{*}\right)$ hold, respectively. Therefore, the mechanism $t(\hat{\boldsymbol{\theta}})$ implements the CIE surplus for $F$ in our model if and only if the mechanism $(q(\hat{\boldsymbol{\theta}}), m(\hat{\boldsymbol{\theta}}))$ efficiently dissolves the partnership $\langle B-D, A-C, F\rangle$. Now, we can apply Propositions 1-3 in CGK and obtain the desired result.

Proposition 1 states that implementability of the CIE surplus diminishes as the agent's incentives to manipulate the bet's outcome become more uneven across states. For an intuition behind this result, consider a mechanism that satisfies $t(a \mid \hat{\boldsymbol{\theta}})-t(b \mid$ $\hat{\boldsymbol{\theta}})=A-C$ if $\hat{\theta}_{1}>\hat{\theta}_{2}$ and $t(a \mid \hat{\boldsymbol{\theta}})-t(b \mid \hat{\boldsymbol{\theta}})=D-B$ if $\hat{\theta}_{1}<\hat{\theta}_{2}$. Then, regardless of the first-period outcome, the agent takes the ex-post efficient action in each state. Moreover, if $\hat{\boldsymbol{\theta}}=\boldsymbol{\theta}$, the bet $t(\hat{\boldsymbol{\theta}})$ is CIE. The problem is to design such a mechanism $t(\hat{\boldsymbol{\theta}})$, which also ensures that the parties participate and report their true priors. To tackle this problem, we reinterpret it as a standard allocation problem. Suppose that both parties report their true priors in period 1. What is the agent's gain in period 2 from choosing the efficient action relative to choosing b? By definition, the gain is zero in state $v$, regardless of whether $\hat{\theta}_{1}$ is higher or lower than $\hat{\theta}_{2}$. However, in state $u$ the gain is $(A-C)-[t(a \mid \hat{\boldsymbol{\theta}})-t(b \mid \hat{\boldsymbol{\theta}})]$. By our construction of $t(\hat{\boldsymbol{\theta}})$ and the assumption that $\hat{\boldsymbol{\theta}}=\boldsymbol{\theta}$, this difference is equal to zero when $\theta_{1}>\theta_{2}$ and equal to $(A-C)+(B-D)$ when $\theta_{1}<\theta_{2}$.

Thus, the agent's gain may be interpreted as a right to receive a prize of $(A-C)+$ $(B-D)$, conditional on choosing $a$ in period 2. Put differently, the right is an asset of size $(A-C)+(B-D)$, whose first-period valuation by the agent is $\theta_{2}[(A-C)+(B-D)]$. Note that the agent receives this asset if and only if $\theta_{2}>\theta_{1}$. This is analogous to allocating the asset to the person who values it the most. If no bet is signed in period 
1 , the agent's gain from choosing the efficient action relative to choosing $b$ is zero in state $v$ and $A-C$ in state $u$. Thus, it is as if the agent initially holds a share of $A-C$ in the asset. His first-period valuation of this asset is $\theta_{2}(A-C)$. By signing the bet with the speculator, the agent increases his share by $B-D$, as long as $\theta_{2}>\theta_{1}$.

These observations suggest that the problem of implementing the CIE surplus is analogous to the problem of dissolving a partnership efficiently. In this problem, two parties jointly hold an asset of size $(A-C)+(B-D)$. The parties' shares in the asset are $A-C$ and $B-D$. Each party privately and independently draws a valuation of the asset. The problem is to design a mechanism that allocates the entire asset to the party with the highest valuation, subject to the constraint that both parties agree to participate in this mechanism. CGK showed that implementing this objective depends on the initial ownership structure. When $A-C \gg B-D$ - that is, if the agent enters the negotiation mostly a "seller" of the asset - the same forces that underlie the Myerson-Satterthwaite theorem make it hard to allocate the asset efficiently. As the gap between $A-C$ and $B-D$ shrinks, each party enters the negotiation both as a seller and a buyer, and thus he has "countervailing incentives" when reporting his valuation. Translated into the language of our model, this result means that implementing the CIE bet becomes easier when the agent's costs of unilaterally manipulating the bet become more even across states.

When $A-C=B-D$, the CIE surplus can be implemented using a natural indirect mechanism. In period 1 , the two parties play a first-price, sealed-bid auction in order to determine who is awarded a prize of $A-C$ (paid by the other party), conditional on the agent choosing $a$ in period 2. The revenues from the auction are equally distributed between the two parties. By translating Propositions 5 and 6 in CGK into the language of our model, we can show that the two-period game induced by this auction implements the CIE surplus for any distribution $F$.

The main message of our example is that when parties with heterogeneous prior beliefs bet on a manipulable outcome, they generate speculative gains in much the same way as when parties with heterogeneous tastes realize gains from trade. In both cases, an efficient allocation assigns an asset to the party who values it the most. In the case of a bet, the "asset" is the right to receive a "prize" in some state, and a party's valuation of this asset is his prior on that state. The size of the asset (i.e., the amount of the prize), as well as the parties' initial claims to the asset, are determined by the costs of unilateral manipulation of the bet's outcome. By implication, these costs also determine the implementability of interim-efficient bets. 


\section{References}

[1] Bazerman, M. H., and J. J. Gillespie (1999): Betting on the Future: The Virtues of Contingent Contracts, Harvard Business Review, 155-160.

[2] Cramton, P., R. Gibbons and P. Klemperer (1987): Dissolving a Partnership Efficiently, Econometrica, 55, 615-632.

[3] Chung, K. and J. Ely (2005): Foundations of Dominant Strategy Mechanisms, Review of Economic Studies, forthcoming.

[4] Eliaz, K. and R. Spiegler (2005): Speculative Contracts, Working Paper, New York University and Tel Aviv University.

[5] Eliaz, K. and R. Spiegler (2006): Contracting with Diversely Naive Agents, Review of Economic Studies 73, 689-714.

[6] Landlier, A. and D. Thesmar (2005): Optimistic Beliefs as Private Benefits for Entrepreneurs: Implications for Contracting and Evidence, Working Paper, New York University and CREST.

[7] Morris, S. (1994): Trade with Heterogeneous Prior Beliefs and Asymmetric Information, Econometrica, 62, 1327-1347.

[8] Myerson, R. and M. Satterthwaite (1983): Efficient Mechanisms for Bilateral Trading, Journal of Economic Theory, 29, 265-281.

[9] Newbery, D. M.G. (1984): Manipulation of Futures Markets by a Dominant Producer, in The Industrial Organization of Futures Markets, ed. by R. W. Anderson. Lexington, MA: Lexington Books. 